Benita Tjoe, MD*

Barbra Streisand Women's Heart Center,

Smidt Heart Institute, Cedars-Sinai Medical

Center, Los Angeles, CA
Lili Barsky, MD*

Barbra Streisand Women's Heart Center

Smidt Heart Institute, Cedars-Sinai Medical

Center, Los Angeles, CA

\section{Janet Wei, MD}

Barbra Streisand Women's Heart Center

Smidt Heart Institute, Cedars-Sinai Medical

Center, Los Angeles, CA; Investigator, Wom-

en's Ischemia Trial to Reduce Events in Nonobstructive CAD (WARRIOR) NCT03417388; Investigator, Women's Ischemia Syndrome Evaluation (WISE) NCT00000554
Babak Azarbal, MD

Smidt Heart Institute, Cedars-Sinai Medical Center, Los Angeles, CA
C. Noel Bairey Merz, MD

Director, Barbra Streisand Women's Heart Center

Smidt Heart Institute, Cedars-Sinai Medical

Center, Los Angeles, CA; Investigator, Women's

Ischemia Trial to Reduce Events in Nonobstruc-

tive CAD (WARRIOR) NCT03417388; Investigator,

Women's Ischemia Syndrome Evaluation (WISE)

NCT00000554
Chrisandra Shufelt, MD, MS

Associate Director, Barbra Streisand Women's Heart Center, Smidt Heart Institute, Cedars-Sinai Medical Center, Los Angeles, CA; Investigator, Women's Ischemia Trial to Reduce Events in

Nonobstructive CAD (WARRIOR) NCT03417388:

Investigator, Women's Ischemia Syndrome Evaluation (WISE) NCT00000554

\title{
Coronary microvascular dysfunction: Considerations for diagnosis and treatment
}

\section{ABSTRACT}

Ischemia and no obstructive coronary artery disease (INOCA) is an increasingly recognized cause of angina, and it is more commonly diagnosed in women. Coronary microvascular dysfunction (CMD), or the abnormal dilation and constriction of the small vessels of the heart, is the underlying cause of INOCA in one-half of cases. This review discusses coronary microvascular pathophysiology, considerations for invasive coronary function testing and noninvasive diagnostic modalities, implications for management, and remaining knowledge gaps.

\section{KEY POINTS}

Women presenting with signs and symptoms of myocardial ischemia are more likely than men to have no obstructive coronary artery disease.

CMD should be considered in patients presenting with persistent angina, evidence of ischemia, and no obstructive coronary artery disease.

CMD is associated with considerable risk of major adverse cardiac events including heart failure, myocardial infarction, stroke, and death.

*These authors contributed equally to this work. doi:10.3949/ccjm.88a.20140
7 rom one-half to two-thirds of women with 4 angina symptoms who undergo coronary angiography for suspected ischemic heart disease have no obstructive coronary artery disease (CAD), according to some estimates. ${ }^{1-3}$ This condition, characterized by signs and symptoms of ischemia in the setting of nonobstructive CAD (defined as $<50 \%$ stenosis on diagnostic angiography), ${ }^{4}$ is termed "ischemia and no obstructive coronary artery disease" (INOCA). ${ }^{5}$ Recent studies have estimated that there are at least 3 to 4 million patients with stable INOCA in the United States, ${ }^{6}$ and it is more prevalent in women than in men. ${ }^{7}$ The overall prevalence of INOCA has been increasing as clinical recognition grows, along with expanded use of diagnostic tests to assess microvascular dysfunction.

Coronary microvascular dysfunction (CMD) and vasospasm of the epicardial arteries are the 2 most common causes of INOCA. ${ }^{8}$ In studies, nearly $50 \%$ of patients with INOCA have been found to have abnormal vasomotor behavior of the coronary microvasculature. ${ }^{9}$ Although CMD occurs in both men and women, it is more prevalent in women, with a 2015 study showing that $66 \%$ of females and $60 \%$ of males with nonobstructive CAD had CMD on invasive testing. ${ }^{10}$

This work was supported by contracts from the National Heart, Lung, and Blood Institutes Nos. N01-HV-68161, N01-HV-68162, N01-HV-68163, N01-HV-68164, grants U0164829, U01 HL649141, U01 HL649241, K23HL105787, K23HL127262, K23HL125941, T32HL69751, R01 HL090957, R01 HL146158, U54 AG065141, 1R03AG032631 from the National Institute on Aging, GCRC grant M01-RR00425 from the National Center for Research Resources, the National Center for Advancing Translational Sciences grants UL1TR000124 and UL1TR000064, and grants from the Gustavus and Louis Pfeiffer Research Foundation, Danville, NJ; The Women's Guild of Cedars-Sinai Medical Center, Los Angeles, CA; The Ladies Hospital Aid Society of Western Pennsylvania, Pittsburgh, PA; QMED Inc, Laurence Harbor, NJ; the Edythe L. Broad and the Constance Austin Women's Heart Research Fellowships, Cedars-Sinai Medical Center, Los Angeles, CA; the Barbra Streisand Women's Cardiovascular Research and Education Program, Cedars-Sinai Medical Center, Los Angeles, CA; The Society for Women's Health Research (SWHR), Washington, DC; and the Linda Joy Pollin Women's Heart Health Program, Erika Glazer Women's Heart Research Initiative, and Adelson Family Foundation, Cedars-Sinai Medical Center, Los Angeles, CA. 
Historically, patients with INOCA were thought to have a good prognosis and relatively low incidence of a major adverse cardiac event (MACE). However, this understanding has since been refuted by studies showing that patients with INOCA have elevated risk for cardiovascular events including acute coronary syndrome, heart failure hospitalization, stroke, and death. ${ }^{6}$ The Women's Ischemia Syndrome Evaluation (WISE) study, started in 1996, has followed more than 900 women with signs and symptoms of myocardial ischemia who had undergone clinically indicated coronary angiography. Of these women, nearly two-thirds were found to have no obstructive CAD. ${ }^{11}$ An analysis of this cohort found that INOCA was associated with a higher rate of MACE than in patients with normal coronary arteries, with a $2.5 \%$ rate of death, nonfatal myocardial infarction, nonfatal stroke, and heart failure hospitalization at the 5-year followup. 5

Hospitalizations for angina were found to have occurred at relatively constant rates during a 9.1-year follow-up study. ${ }^{11}$ Furthermore, women with stable INOCA and nonobstructive $\mathrm{CAD}$ had 10-year all-cause mortality and cardiac mortality rates of $17 \%$ and $11 \%$, respectively, compared with $10 \%$ and $6 \%$ in women with normal coronary arteries. ${ }^{12}$ The WISE study has also shown that females with INOCA are at increased risk for progression to obstructive CAD. ${ }^{13}$

In addition to adverse clinical outcomes, CMD and persistent anginal symptoms affect patients' quality of life, limit their exercise capacity, and may contribute to unnecessary testing, costs, and more frequent healthcare visits. ${ }^{14}$ Thus, early diagnosis and intervention are crucial to improving outcomes.

At present, invasive coronary function testing (CFT) is the technical standard for diagnosing CMD and coronary vasospasm. It can identify those at higher risk of MACE and help facilitate medical management. This review will discuss coronary microvasculature physiology, CFT, noninvasive diagnostic modalities, and treatment options, as well as knowledge gaps and future directions regarding CMD.

\section{TINY VESSELS, BIG EFFECTS}

The coronary microvasculature consists of the smaller cardiac vessels including the pre-arterioles (diameter 100-500 $\mu \mathrm{m}$ ) and intracardiac arterioles $(<100 \mu \mathrm{m}) .{ }^{15}$ These arterioles are regulated by different mechanisms (Figure 1) that work in tandem to modulate cardiac blood flow, as follows:

- Larger proximal arterioles use endothelialdependent vasodilatory mechanisms by which an increase in coronary blood flow leads to vasodilation and a decrease in blood flow leads to vasoconstriction

- Medium-sized arterioles have vascular smooth muscle cell stretch receptors to detect intraluminal pressure

- The smallest distal arterioles are regulated by local metabolic activity. ${ }^{15}$

The endothelium, or the layer of cells lining the arteries and arterioles, plays a vital role in regulating blood flow to the myocardium. ${ }^{16}$ A healthy endothelium promotes vasodilation, antioxidant effects, inhibition of smooth muscle cell proliferation, and anticoagulant effects. Furthermore, endothelial cells act to regulate inflammation and serve as a barrier to potentially toxic materials. ${ }^{17}$ An imbalance of nitric oxide consumption is thought to be the primary driver of dysfunction, leading to the inability to properly dilate and to subsequent ischemia. ${ }^{18}$ Endothelium dysfunction is a principal contributor to both macro- and microvascular coronary dysfunction and is thought to be a key player in the development of plaque progression and atherosclerosis. ${ }^{16}$

CMD may be characterized by heightened sensitivity of the small vessels to vasoconstrictor stimuli and decreased microvascular vasodilator capacity. ${ }^{19}$ In healthy vessels, adenosine, acetylcholine, and nitroglycerin induce vasodilation. ${ }^{20}$ However, in patients with CMD, the microvasculature may exhibit a blunted vasodilatory response to these agents. Coronary flow reserve (CFR) - the ratio of coronary blood flow at maximal dilation in response to intracoronary adenosine from baseline-is impaired in patients with CMD. In the setting of endothelial dysfunction, acetylcholine may induce paradoxical vasoconstriction and micro- or macrovascular vasospasm in compromised vessels. Nitroglyc- 


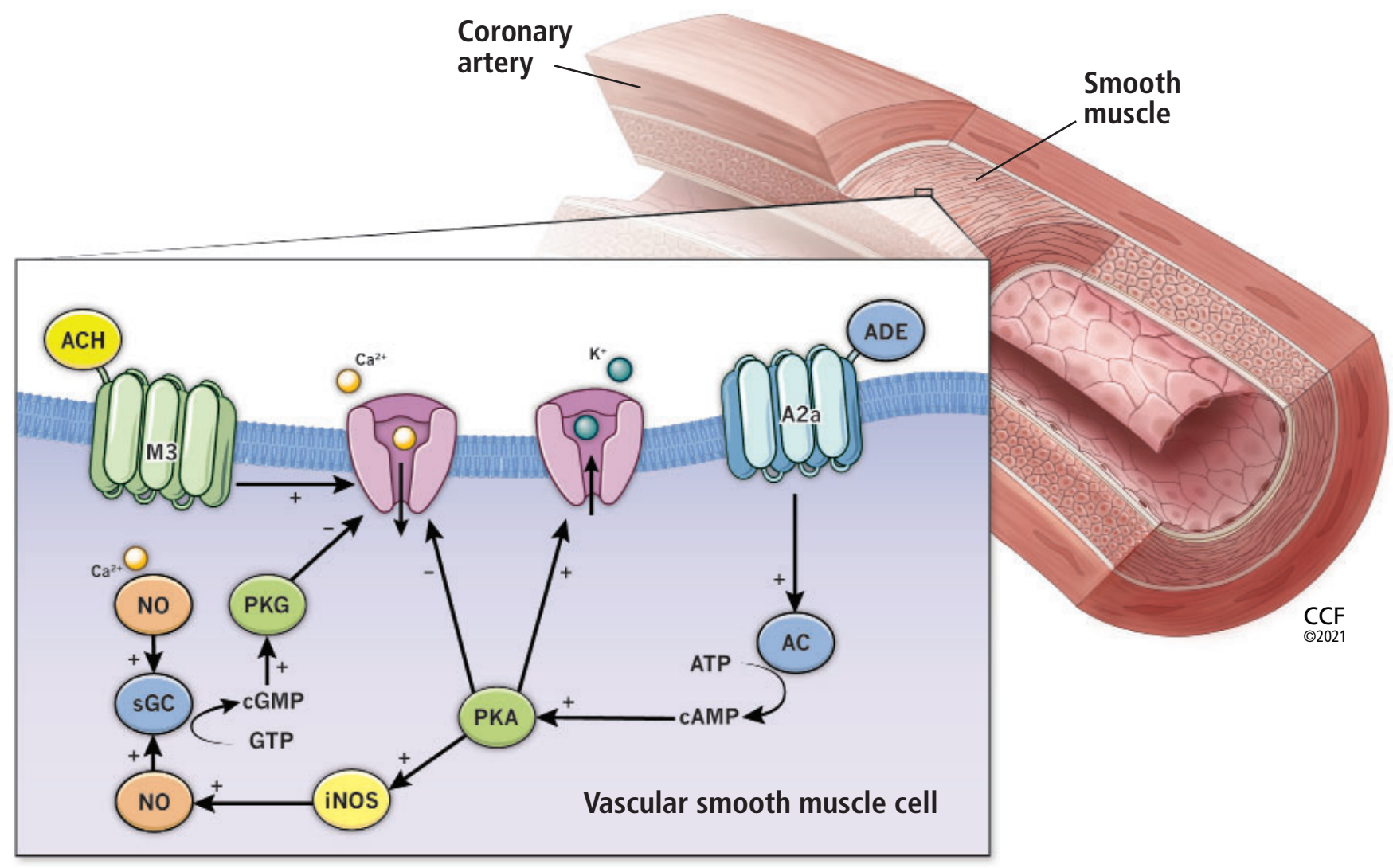

Figure 1. The effects of acetylcholine $(A C H)$ and adenosine (ADE) on the smooth muscle of the coronary vasculature. ACH binds to the muscarinic receptor (M3), stimulating the release of calcium $\left(\mathrm{Ca}^{2+}\right)$ into the vascular smooth muscle cell, which drives nitric oxide (NO) formation for vasodilation and also drives contraction for vasoconstriction. ADE stimulates the conversion of adenosine triphosphate (ATP) to cyclic adenosine monophosphate (cAMP), leading to inhibition of calcium influx and induction of nitric oxide formation, both of which result in vasodilation.

$\mathrm{A} 2 \mathrm{a}=$ adenosine receptor; $\mathrm{AC}=$ adenylate cyclase; $\mathrm{ACh}=$ acetylcholine; $\mathrm{ADE}=$ adenosine; $\mathrm{ATP}=$ adenosine triphosphate; $\mathrm{Ca}^{2+}=$ calcium; CAMP = cyclic adenosine monophosphate; cGMP = cyclic guanosine monophosphate; eNOS = endothelial nitric oxide synthase; GTP = guanosine triphosphate; iNOS = inducible nitric oxide synthase; $\mathrm{M} 3=$ muscarinic receptor; $\mathrm{NO}=$ nitric oxide; $\mathrm{PKA}=$ protein kinase activation; PKG = guanosine monophosphate-dependent protein kinase; sGC = soluble guanylate-cyclase

erin response is used to evaluate for nonendothelial-dependent macrovascular function.

\section{RISK FACTORS AND CLINICAL PRESENTA- TION OF MICROVASCULAR DYSFUNCTION}

Traditional cardiovascular risk factors, including hypertension, hyperlipidemia, advanced age, obesity, smoking, and diabetes, have been found to be associated with CMD. ${ }^{19,21}$ Aging is associated with an increase in arterial wall stiffness, medial thickening, and lumen en- largement that results in an increase in pulse pressure and hypertrophy of arteries, ultimately contributing to endothelial dysfunction..$^{22}$ Studies have found that CFR is reduced in patients with diabetes, which is thought to be a consequence of the microvascular inflammation that also leads to diabetic retinopathy and nephropathy. ${ }^{23}$ Additionally, smokers and patients with chronic inflammatory conditions such as rheumatoid arthritis and systemic lupus erythematosus often have lower CFR, with a $21 \%$ reduction observed in smokers. ${ }^{24}$ 


\section{Coronary function testing: Protocol and methods}

Several protocols exist for coronary function testing for the purposes of evaluating for coronary microvascular dysfunction (CMD) and coronary vasospasm. At our institution, we utilize the following methods:

Patient preparation. Patients fast for 12 hours before the scheduled procedure..$^{30}$ To avoid confounding of results, patients are asked to discontinue caffeine, long-acting nitrates, short-acting calcium channel blockers, alpha- and beta-blockers, angiotensinconverting enzyme inhibitors, angiotensin receptor blockers, renin and aldosterone inhibitors, ranolazine, diuretics, angiotensinneprilysin inhibitors, ticagrelor, and aspirin-dipyridamole for 24 hours before the procedure. Long-acting calcium channel blockers are withheld for 48 hours, and sublingual nitroglycerin and nicotine are held for 4 hours prior to testing.

Vasoactive agent preparation. Two concentrations of acetylcholine $(0.182 \mu \mathrm{g} / \mathrm{mL}$ and $18.2 \mu \mathrm{g} / \mathrm{mL})$ are premixed by the hospital pharmacy, within 3 hours of the scheduled procedure. ${ }^{30}$ Two doses of adenosine $(18 \mu \mathrm{g}$ and $100 \mu \mathrm{g})$ and $200 \mu \mathrm{g}$ of nitroglycerin are prepared by the catheterization laboratory nursing staff. Of note, the precise agent dosages used may vary by institution.

Angiography to confirm nonobstructive disease. Coronary angiography is performed to assess for atherosclerosis, myocardial bridging, anomalies, and slow flow. Any catheter-induced spasm or chest pain observed during contrast administration or catheter manipulation is also documented. Left ventricular end-diastolic pressure is measured. Fractional flow reserve should be measured to rule out hemodynamically significant stenoses. Any obstructive disease or spontaneous spasm observed at this time may obviate the need for further testing.

Intracoronary vasoactive agent infusions. Patients are given body-weight-adjusted heparin to achieve activated clotting time above 250 seconds..$^{30} \mathrm{~A} 0.014$-inch Doppler guidewire is placed into the proximal to mid left anterior descending artery until an adequate Doppler signal is obtained. Prior to each infusion, a baseline heart rate, blood pressure, and average peak velocity of blood flow are recorded. At specified time intervals, the peak heart rate, blood pressure, and average peak velocity are documented. The line is flushed, and return to baseline average peak velocity is permitted before infusion of subsequent dosages and agents. Any symptoms, ischemic changes on electrocardiography, or arrhythmias during infusions are noted. Cine images are taken at 2 time points: when the baseline average peak velocity is measured, and immediately after the peak average peak velocity is measured. This allows quantitative coronary angiography measurement of the vessel diameter $5 \mathrm{~mm}$ distal to the tip of the Doppler guidewire.

Assessment of mechanistic pathways and significance. This assessment is most often performed to completion in the following order to assess for the simultaneous presence of multiple pathways of coronary artery dysfunction. If the patient experiences a complication at any time, the procedure may be aborted.

1. Nonendothelial dependent microvascular function: First, $18-\mu \mathrm{g}$ and $100-\mu \mathrm{g}$ dosages of adenosine are consecutively delivered via intracoronary bolus to induce maximal hyperemia. ${ }^{30}$ Coronary flow reserve is calculated as the ratio of the peak to baseline average peak velocities. ${ }^{30,31} \mathrm{~A}$ peak flow reserve $<2.5$ is considered abnormal.

2. Endothelial-dependent macrovascular function: Next, the $0.182-\mu \mathrm{g} / \mathrm{mL}$ and $18.2-\mu \mathrm{g} / \mathrm{mL}$ concentrations of acetylcholine are administered via infusion pump at a rate of 2 to $3 \mathrm{~mL}$ over 3 minutes. The change in vessel diameter is then calculated by quantitative coronary angiography. An increase in mean lumen diameter of $\leq 5 \%$ suggests dysfunction. Of note, the infusion rate may vary according to institutional protocol. Coronary angiography is performed after each dosage of acetylcholine to evaluate for epicardial vasospasm. It is important to withdraw the contents of the guide catheter prior to angiography in order to avoid inadvertently delivering a bolus of any acetylcholine that may be left in the guide catheter after the slow infusion.

3. Endothelial-dependent microvascular function: The aforementioned infusion of the $18.2-\mu \mathrm{g} / \mathrm{mL}$ concentration of acetylcholine is used to calculate coronary blood flow, with 2 to $3 \mathrm{~mL}$ infused over 3 minutes. Coronary angiography is performed after each dose of acetylcholine to evaluate for epicardial vasospasm. Again, it is important to withdraw the contents of the guide catheter prior to angiography. A $<50 \%$ increase in coronary blood flow is considered abnormal. As described above, the infusion rate may vary according to institutional protocol.

4. Vasospasm: As the graded $0.182-\mu \mathrm{g} / \mathrm{mL}$ and $18.2-\mu \mathrm{g} / \mathrm{mL}$ dose infusions of acetylcholine are given, the operator assesses for signs of vasospasm. If no spasm is exhibited, a higher concentration of acetylcholine is infused at a rate of $2 \mathrm{~mL}$ per minute for 3 minutes. If spasm is provoked, subsequent higher acetylcholine dose testing is withheld, and nitroglycerin is administered immediately. Epicardial vasospasm is defined as a diameter reduction $>90 \%$ associated with chest pain or ischemic ST-segment changes, or both. Microvascular coronary spasm is suggested by chest pain and ischemic ST-segment changes without significant epicardial artery vasoconstriction.

5. Nonendothelial-dependent macrovascular function: After acetylcholine infusion, nitroglycerin (150-200 $\mu \mathrm{g})$ is given, and quantitative coronary angiography is performed after 30 seconds. Dilation $<20 \%$ is considered abnormal. This measurement allows for assessment of the macrovascular responsiveness to treatment with nitrates.

6. Nociceptive abnormality: Heightened pain sensitivity at any point in the procedure, demonstrated by chest pain during catheter manipulation or contrast administration, is suggestive of nociceptive abnormality. 
There is also evidence that women with a history of an adverse pregnancy outcome such as preeclampsia, gestational hypertension, or diabetes may have an increased risk of CMD. ${ }^{13}$

Symptoms include chest discomfort, dyspnea, and reduced exercise tolerance, with some patients having angina that persists after cessation of exertion. ${ }^{25}$ Because nitroglycerin acts preferentially to dilate larger vessels of the heart and has little effect on the smaller arterioles, it may not provide symptom relief to patients with $\mathrm{CMD} .{ }^{26}$ Objective clinical evidence of myocardial ischemia can include elevated troponin levels and ST-segment electrocardiographic or imaging abnormalities at rest or with stress. ${ }^{27}$

\section{CORONARY FUNCTION TESTING}

\section{When is it appropriate?}

Testing for CMD should be considered for patients with persistent symptoms and objective signs of myocardial ischemia despite absence of obstructive CAD $(<50 \%$ coronary artery diameter reduction). ${ }^{28}$ But before invasive testing, patients should be evaluated for other diagnoses, including hyperthyroidism, anemia, hypertensive urgency, and substance abuse.

For patients with persistent symptoms but angiographically normal coronary arteries or nonobstructive CAD, the 2019 European Society of Cardiology guidelines recommend "consideration" (ie, the weight of evidence favors efficacy, but it is not well established) of guidewire-based CFR measurements and intracoronary acetylcholine for assessment of spasm. ${ }^{29}$ These guidelines also recommend consideration of noninvasive transthoracic Doppler of the left anterior descending artery, cardiac magnetic resonance imaging, and positron emission tomography for CFR measurement. ${ }^{29}$

The decision to proceed with invasive CFT depends on a variety of factors including local hospital practices, patient preference, goals, and availability of CFT and noninvasive diagnostic modalities. Discussion with the patient includes a comprehensive individualized evaluation to determine whether there is more benefit than risk. In patients with prior myocardial infarction or high suspicion for vasospasm, invasive CFT with acetylcholine provocation testing is preferable based on its ability to delineate pathways and elicit spasm.

For a detailed description of the CFT protocol used at our institution, see "Coronary function testing: Protocol and methods." 30,31

\section{Procedure}

CFT is an angiographic procedure to evaluate both endothelial-dependent and nonendothelial-dependent macrovascular and microvascular response to vasoactive agents in patients with INOCA (Table 1). After diagnostic angiography to exclude obstructive epicardial disease, myocardial bridging, and other coronary anomalies, the drugs adenosine, acetylcholine, and nitroglycerin are administered sequentially to evaluate for microvascular function, vasospasm, and smooth muscle response. ${ }^{32,33}$

Functional testing involves inserting a guiding catheter and positioning a Doppler wire into the coronary artery to be studied. Typically, this evaluation is done in the left anterior descending artery, but it can also be performed in the left circumflex and right coronary arteries.

Adenosine. First, an intracoronary injection of adenosine is given to assess nonendothelial-dependent dysfunction. Flow reserves are measured before and after adenosine administration. Graded doses are used to achieve maximum hyperemia. A CFR below 2.5 is diagnostic for nonendothelial-dependent microvascular dysfunction. When interpreting CFR results, it is important to note that this measurement has been shown to be a continuous predictor of MACE, similar to blood pressure and low-density lipoprotein cholesterol levels. ${ }^{5}$ It has also been shown that patients with stable INOCA and a CFR below 2.0 experience higher MACE rates (in females and males). ${ }^{34}$ In the WISE study, it was shown that a CFR below 2.32 best predicted adverse outcomes in women. ${ }^{5}$

Acetylcholine. Next, acetylcholine is administered to assess for endothelial-dependent dysfunction. It is given in increasing concentrations to stimulate the release of nitric oxide. An increase in coronary blood flow less than $50 \%$ is diagnostic of endothelialdependent microvascular dysfunction. Vessel diameter response is measured, and a change of less than $5 \%$ is diagnostic for endothelialdependent macrovascular dysfunction. A

\section{Coronary microvascular dysfunction is more prevalent in women}




\section{TABLE 1}

\section{Invasive coronary function testing in patients with INOCA: Three medications}

Drug administered

1. Adenosine

2. Acetylcholine

3. Nitroglycerin
Results

Coronary flow reserve $<2.5$

$<50 \%$ increase in coronary blood flow

$<5 \%$ increase in coronary artery diameter

$>90 \%$ decrease in coronary artery diameter

Chest pain and ischemic ST-segment changes on electrocardiography

Chest pain and ischemic ST-segment changes on electrocardiography in the absence of significant epicardial coronary vasoconstriction

$<20 \%$ increase in coronary artery diameter
Diagnosis

Nonendothelial-dependent microvascular dysfunction

Endothelial-dependent microvascular dysfunction

Endothelial-dependent macrovascular dysfunction

Epicardial coronary spasm

Microvascular coronary spasm dysfunction
Endothelial dysfunction contributes to macro- and microvascular coronary dysfunction

INOCA = ischemia and no obstructive coronary artery disease (ie, $<50 \%$ stenosis)

higher dose of acetylcholine is given to assess for coronary spasm. Coronary vasospasm is defined as more than a $90 \%$ diameter reduction with chest pain and ischemic ST-segment changes on electrocardiography.

Nitroglycerin. The last drug administered is nitroglycerin to test macrovascular function. A change in diameter of less than $20 \%$ indicates abnormal smooth muscle reactivity and nonendothelial-dependent macrovascular dysfunction.

If the patient experiences chest pain during catheter manipulation or contrast administration, it suggests nociceptive abnormality. This is thought to be associated with altered afferent neuronal pathways, change in cerebral cortical activation, or reduced endogenous opioid release. ${ }^{28}$

CFT has been shown to be safe and effective overall, with the WISE study reporting a low rate of serious adverse events $(0.7 \%),{ }^{32}$ although these rates were observed at healthcare centers of excellence. A prospective multicenter study has reported cases of coronary artery dissection, ST-elevation myocardial infarction associated with vasospasm, transient air microembolism, and deep vein thrombosis, but the overall rate of periprocedural adverse events was low at $1.4 \% .{ }^{32}$ As the reactivity testing prolongs the length of angiography, precautions must be taken for those at higher risk of contrast-induced nephropathy. ${ }^{35}$ Adenosine should be avoided or used with caution in patients with a history of asthma, as it may contribute to bronchospasm.

\section{OTHER DIAGNOSTIC APPROACHES}

Pharmacologic stress testing using noninvasive diagnostic modalities-positron emission tomography, cardiac magnetic resonance imaging, and transthoracic Doppler echocardiography - and an empiric approach to therapy 
can also be used to assess for CMD. These may provide a suitable approach for patients who are hesitant to proceed with invasive testing.

Positron emission tomography utilizes various radioactive tracers, in patients both at rest and with vasodilator-induced stress, to quantify absolute myocardial blood flow and detect regional variations suggestive of CMD. Computed tomography can also be performed to determine the coronary artery calcium score for risk stratification. ${ }^{33}$

Cardiac magnetic resonance imaging is a tool with high diagnostic accuracy, low ionizing radiation, and high spatial resolution. It can be used to quantify the myocardial perfusion reserve index and assess for late gadolinium enhancement, a signal of myocardial damage and scar associated with vasomotor dysfunction. ${ }^{36}$ The presence of scar is useful for risk stratification. This modality is more widely available than invasive CFT.

Doppler echocardiography of the left anterior descending artery can be used to quantify coronary blood flow. CFR calculated by this procedure has been shown to correlate well with measurements obtained through positron emission tomography and invasive techniques. ${ }^{30}$ This method is less expensive and more accessible than other techniques and lacks ionizing radiation, but limits evaluation to that of the left anterior descending artery.

Empiric therapy is an approach that assesses the cardiac response to an empiric trial of drug therapy. For example, in patients with sporadic angina responsive to nitrates suggestive of vasospasm, providers can implement and monitor symptomatic response to a trial of calcium channel blockers. For those with comorbid hyperlipidemia and hypertension and high pretest probability of endothelial dysfunction and CMD, the anginal response to statins and angiotensin-converting enzyme (ACE) inhibitors can be monitored. This approach may be suitable for patients with contraindications or allergies precluding diagnostic procedures.

\section{DRUG AND NONDRUG THERAPIES}

Using CFT to identify affected pathways helps guide selection of the best targeted therapies (Table 2). It is important to note that treatment strategies are not clear or standardized,

\section{TABLE 2}

\section{Treatments for coronary microvascular dysfunction based on the pathway identified by invasive coronary function testing}

\author{
Endothelial dysfunction \\ Angiotensin-converting enzyme inhibitor \\ Angiotensin receptor blocker \\ Statin \\ L-arginine \\ Cardiac rehabilitation
}

Enhanced external counterpulsation

Nonendothelial dysfunction

Angiotensin-converting enzyme inhibitor

Beta-blocker

Alpha-/beta-blocker

Ranolazine

Ivabradine

Phosphodiesterase- 5 inhibitor

Vasospasm

Calcium channel blocker

Nitrate

Nociceptive abnormality

Tricyclic antidepressant

Spinal cord stimulation

Cognitive behavior therapy

largely because of a lack of evidence-based guidelines, but there is some evidence to follow.

\section{Nonendothelial-dependent dysfunction}

Nonendothelial CMD is treated with drugs targeting ischemia. Beta-blockers and betablockers with alpha-blocking activity reduce the frequency and severity of angina and improve CFR. ${ }^{37}$ They act by reducing myocardial oxygen consumption and increasing diastolic filling time. Both short-acting and long-acting nitrates help angina by promoting vasodilation and reducing preload. ${ }^{37}$ Ranolazine and ivabradine can be considered in patients with

\section{Consider}

coronary

function testing when myocardial ischemia is suspected in the presence of nonobstructive CAD 
refractory angina and contraindications to traditional antianginal drugs as they have less hemodynamic effect. More recently, phosphodiesterase- 5 inhibitors have been used to drive vasodilation in CMD.

\section{Endothelial dysfunction}

Treatment options aimed at both macro- and microvascular dysfunction are similar to those targeting atherosclerotic disease, including ACE inhibitors, angiotensin receptor blockers, statins, and low-dose aspirin. High-dose quinapril has been associated with reduced angina in women with CMD, likely by reducing vascular inflammation. ${ }^{38}$ Statins have been shown to improve exercise tolerance and reduce angina due to their anti-inflammatory effects on endothelial function. ${ }^{39}$ Aspirin is recommended based on the observation that even if no significant plaque burden is seen on angiography, most patients with CMD have coronary atherosclerosis when evaluated by intravascular ultrasonography. ${ }^{40} \mathrm{~L}$-arginine, a precursor of nitric oxide, has been shown to improve coronary blood flow. ${ }^{41}$

\section{Vasospasm}

Calcium channel blockers and nitrates are Pharmacologic stress testing with noninvasive diagnostic modalities is an alternative to invasive testing preferred in the setting of vasospastic angina. ${ }^{29}$ The calcium channel blockers recommended are amlodipine, diltiazem, verapamil, and long-acting nifedipine. A randomized trial of patients with angina, small coronary arteries, and limited vasodilator reserve showed that patients on verapamil and nifedipine had fewer episodes of angina, consumed fewer nitroglycerin tablets, and had greater exercise tolerance than patients on placebo. ${ }^{42}$ Tachyphylaxis is a known risk with long-term nitrate use and, thus, drug-free intervals (12 hours daily) are recommended. Unopposed beta-blockade should be avoided as it may contribute to coronary artery spasm. ${ }^{43}$ If betablockers are indicated in patients with vasospasm, a combined alpha-beta agent such as carvedilol is favored.

\section{Nociceptive abnormality}

For nociceptive abnormality identified by CFT, low-dose tricyclic antidepressants reduce the frequency of angina. In refractory cases, spinal cord stimulation, cognitive behavior therapy, and biofeedback can be considered. ${ }^{37}$

\section{Nonpharmacologic treatments}

Several nonpharmacologic approaches are available for CMD. Cardiac rehabilitation has been shown to improve diastolic resting blood pressure, body mass index, and exercise capacity. These programs also improve overall quality of life and psychological morbidity. ${ }^{37,44}$ Enhanced external counterpulsation, a therapy consisting of pneumatic stockings on the lower extremities electronically timed to inflate during diastole and deflate during systole, can also improve CMD symptoms. ${ }^{37}$ This therapy promotes collateral coronary flow and improves endothelial function by reducing afterload and increasing preload. Therapeutic lifestyle recommendations include smoking cessation, nutrition counseling, weight reduction, and regular, moderate-intensity physical therapy.

\section{KNOWLEDGE GAPS REMAIN}

Despite considerable evidence regarding MACE and long-term adverse event prognosis associated with INOCA, neither the American College of Cardiology nor the American Heart Association has guidelines for therapy. As a result, internists and cardiologists may lack confidence in the recognition, diagnosis, and management of this phenotype of ischemic heart disease. In 2017, the American College of Cardiology convened a group to review the current knowledge and provide next steps for evidence-based management. ${ }^{5}$ In 2019, the European Society of Cardiology published guidelines for chronic coronary syndromes that include a discussion and recommendation for evaluation of vasospastic and microvascular disease. ${ }^{29}$

At present, no standardized diagnostic algorithm exists. Decisions regarding testing may depend on patient risk stratification (ie, history of prior myocardial infarction), the impact of anginal symptoms on quality of life, and local availability of testing modalities. Compared with noninvasive methods, functional reactivity testing has the benefit of identifying the specific mechanism of dysfunction (ie, nonendothelial-dependent vs endothelial-dependent, presence of spasm, and nociceptive abnormality) to better direct therapy. However, this approach is invasive, 
can be time-consuming, and requires specially trained cardiac interventionalists. ${ }^{27}$ Ongoing investigations are evaluating how functional testing may be implemented following standard diagnostic angiography when no obstructive lesions are found. A streamlined and abbreviated protocol of adenosine for CFR measurement followed by acetylcholine to observe angiographically for vasospasm can be easily used by interventionalists.

In addition, differences of INOCA in males vs females remain evident and require further investigation. One hypothesis is that women may have a greater ability to widen and narrow arteries perhaps as a result of the need to control blood flow during pregnancy. Additionally, women have been shown to have more pain sensation than men and, thus, may have more perceived pain and anginal symptoms. ${ }^{45}$ With regard to outcomes, females with INOCA have been shown to have higher rates of cardiac events than males. One study of 13,695 patients with INOCA found a 3-fold higher MACE rate in women compared with men in the first year. ${ }^{46} \mathrm{~A}$ significant knowledge gap remains on this matter.

\section{FUTURE DIRECTIONS}

Recent studies have suggested that cardiomyocyte injury and myocardial stiffness caused by CMD play a role in the pathophysiology of patients with heart failure and preserved ejection fraction (HFpEF). ${ }^{47}$ It is hypothesized that microvascular endothelial dysfunction, decreased nitric oxide bioavailability, and increased cytokine signaling may contribute to the increased microvascular inflammation and myocardial fibrosis observed in these patients. ${ }^{48,49}$ Clinically, decreased CFR has been found to be associated with diastolic dysfunction and with a 5-fold increased rate of hospitalizations for $\mathrm{HFpEF}^{47}$ Clinical studies also suggest a higher prevalence of $\mathrm{HFpEF}$ in women than in men. ${ }^{50}$ The association between CMD and HFpEF needs further investigation, specifically with regard to sex differences.

To better target prevention and treatment, practitioners need to understand the risk factors. While traditional comorbid risk factors such as hypertension, hyperlipidemia, and diabetes have been implicated, less is known about autoimmune conditions and adverse pregnancy outcomes. Additionally, much remains to be studied about novel risk markers including high-sensitivity C-reactive protein, lipoproteins, interleukins, and other inflammatory markers.

Regarding treatment, more randomized trials are needed to guide an evidence-based approach. The Women's Ischemia Trial to Reduce Events in Nonobstructive CAD (WARRIOR), an ongoing multicenter, prospective, randomized, and blinded outcome trial, is designed to explore the long-term outcomes of intensive statin, ACE inhibitor, and aspirin therapy vs usual care in symptomatic women with INOCA. ${ }^{51}$ This trial aims to enroll 4,442 participants. Randomized stem cell trials are also under way to further evaluate the effectiveness of coronary CD34+ infusions in improving CFR in patients with CMD and persistent refractory angina after a trial showed that this treatment effectively reduced hospitalizations, cardiac procedures, and healthcare expenditures in patients with refractory angina..$^{52}$

\section{HOPE FOR STRONG GUIDELINES LIES IN ONGOING CLINICAL TRIALS}

In patients who present with angina symptoms but who have no evidence of obstructive coronary artery disease, it is important to consider the diagnoses of INOCA and CMD given the substantial morbidity associated with this condition. At present, there is no uniform comprehensive diagnostic and therapeutic strategy or algorithm, but several options exist. Both noninvasive and invasive tests are available to establish the diagnosis. CFT is currently the therapeutic standard, providing a means to determine the mechanistic pathways for CMD that, in turn, guide targeted therapeutic options with the goal of preventing future adverse cardiac events. Our evolving knowledge of CMD and its management relies on ongoing investigations and outcomes of clinical trials.

\section{DISCLOSURES}

Dr. Wei has disclosed membership on advisory committees or review panels for Abbott Vascular. Dr. Bairey Merz has disclosed consulting for Abbott Diagnostic and Sanofi and board membership for iRhythm. The other authors report no relevant financial relationships which, in the context of their contributions, could be perceived as a potential conflict of interest. 


\section{CORONARY MICROVASCULAR DYSFUNCTION}

\section{REFERENCES}

1. Farrehi PM, Bernstein SJ, Rasak M, et al. Frequency of negative coronary arteriographic findings in patients with chest pain is related to community practice patterns. Am J Manag Care 2002; 8(7):643-648. pmid:12125804

2. Sharaf BL, Pepine CJ, Kerensky RA, et al. Detailed angiographic analysis of women with suspected ischemic chest pain (pilot phase data from the NHLBI-sponsored Women's Ischemia Syndrome Evaluation [WISE] Study Angiographic Core Laboratory). Am J Cardiol 2001; 87(8):937-941. doi:10.1016/s0002-9149(01)01424-2

3. Anderson RD, Petersen JW, Mehta PK, et al. Prevalence of coronary endothelial and microvascular dysfunction in women with symptoms of ischemia and no obstructive coronary artery disease is confirmed by a new cohort: the NHLBI-sponsored Women's Ischemia Syndrome Evaluation-Coronary Vascular Dysfunction (WISE-CVD). J Interv Cardiol 2019; 2019:7169275. doi:10.1155/2019/7169275

4. Ong P, Camici PG, Beltrame JF, et al. International standardization of diagnostic criteria for microvascular angina. Int J Cardiol 2018; 250:16-20. doi:10.1016/j.ijcard.2017.08.068

5. Bairey Merz CN, Pepine CJ, Walsh MN, Fleg JL. Ischemia and no obstructive coronary artery disease (INOCA): developing evidencebased therapies and research agenda for the next decade. Circulation 2017; 135(11):1075-1092. doi:10.1161/CIRCULATIONAHA.116.024534

6. Herscovici R, Sedlak T, Wei J, Pepine CJ, Handberg E, Bairey Merz CN. Ischemia and no obstructive coronary artery disease (INOCA ): what is the risk? J Am Heart Assoc 2018; 7(17):e008868. doi:10.1161/JAHA.118.008868

7. Jespersen L, Abildstrøm SZ, Hvelplund A, et al. Symptoms of angina pectoris increase the probability of disability pension and premature exit from the workforce even in the absence of obstructive coronary artery disease. Eur Heart J 2013; 34(42):3294-3303.

doi:10.1093/eurheartj/eht395

8. Ford TJ, Berry C. How to diagnose and manage angina without obstructive coronary artery disease: lessons from the British Heart Foundation CorMicA trial. Interv Cardiol 2019; 14(2):76-82. doi:10.15420/icr.2019.04.R1

9. Reis SE, Holubkov R, Conrad Smith AJ, et al; WISE Investigators. Coronary microvascular dysfunction is highly prevalent in women with chest pain in the absence of coronary artery disease: results from the NHLBI WISE study. Am Heart J 2001; 141(5):735-741. doi:10.1067/mhj.2001.114198

10. Sara JD, Widmer RJ, Matsuzawa Y, Lennon RJ, Lerman LO, Lerman A. Prevalence of coronary microvascular dysfunction among patients with chest pain and nonobstructive coronary artery disease. JACC Cardiovasc Interv 2015; 8(11):1445-1453. doi:10.1016/j.jcin.2015.06.017

11. Aldiwani H, Zaya M, Suppogu N, et al. Angina hospitalization rates in women with signs and symptoms of ischemia but no obstructive coronary artery disease: a report from the WISE (Women's Ischemia Syndrome Evaluation) study. J Am Heart Assoc 2020; 9(4):e013168. doi:10.1161/JAHA.119.013168

12. Kenkre TS, Malhotra P, Johnson BD, et al. Ten-year mortality in the WISE study (Women's Ischemia Syndrome Evaluation). Circ Cardiovasc Qual Outcomes. 2017; 10(12):e003863. doi:10.1161/CIRCOUTCOMES.116.003863

13. Patel H, Aggarwal NT, Rao A, et al. Microvascular disease and smallvessel disease: the nexus of multiple diseases of women. J Womens Health (Larchmt) 2020; 29(6):770-779. doi:10.1089/jwh.2019.7826

14. Shaw LJ, Merz CN, Pepine CJ, et al. The economic burden of angina in women with suspected ischemic heart disease: results from the National Institutes of Health-National Heart, Lung, and Blood Institute-sponsored women's ischemia syndrome evaluation. Circulation 2006; 114(9):894-904. doi:10.1161/CIRCULATIONAHA.105.609990

15. Kuo L, Davis MJ, Chilian WM. Longitudinal gradients for endothelium-dependent and -independent vascular responses in the coronary microcirculation. Circulation 1995; 92(3):518-525. doi:10.1161/01.cir.92.3.518
16. Lerman A, Burnett JC Jr. Intact and altered endothelium in regulation of vasomotion. Circulation 1992; 86(6 suppl):III12-III19. pmid:1424046

17. Kinlay S, Ganz P. Role of endothelial dysfunction in coronary artery disease and implications for therapy. Am J Cardiol 1997; 80(9A):11116I. doi:10.1016/s0002-9149(97)00793-5

18. Yang Z, Li J, Kong J, Wu S. Impairment of vascular endothelial function following reperfusion therapy in patients with acute myocardial infarction. J Int Med Res 2013; 41(4):1074-1078. doi:10.1177/0300060513487650

19. Chen C, Wei J, AlBadri A, Zarrini P, Bairey Merz CN. Coronary microvascular dysfunction-epidemiology, pathogenesis, prognosis, diagnosis, risk factors and therapy. Circ J 2016; 81(1):3-11. doi:10.1253/circj.CJ-16-1002

20. Britten MB, Zeiher AM, Schächinger V. Clinical importance of coronary endothelial vasodilator dysfunction and therapeutic options. $J$ Intern Med 1999; 245(4):315-327 doi:10.1046/j.1365-2796.1999.00449.x

21. Lee DH, Youn HJ, Choi YS, et al. Coronary flow reserve is a comprehensive indicator of cardiovascular risk factors in subjects with chest pain and normal coronary angiogram. Circ J 2010; 74(7):1405-1414. doi:10.1253/circj.cj-09-0897

22. Moreau $\mathbf{P}$, d'Uscio LV, Lüscher TF. Structure and reactivity of small arteries in aging. Cardiovasc Res 1998; 37(1):247-253. doi:10.1016/s0008-6363(97)00225-3

23. Pitkänen OP, Nuutila $P$, Raitakari OT, et al. Coronary flow reserve is reduced in young men with IDDM. Diabetes 1998; 47(2):248-254. doi:10.2337/diab.47.2.248

24. Kaufmann PA, Gnecchi-Ruscone T, di Terlizzi M, Schäfers KP, Lüsche TF, Camici PG. Coronary heart disease in smokers: vitamin C restores coronary microcirculatory function. Circulation 2000; 102(11):12331238. doi:10.1161/01.cir.102.11.1233

25. Lanza GA, Crea F. Primary coronary microvascular dysfunction: clinical presentation, pathophysiology, and management. Circulation 2010; 121(21):2317-2325. doi:10.1161/CIRCULATIONAHA.109.900191

26. Kanatsuka H, Eastham CL, Marcus ML, Lamping KG. Effects of nitroglycerin on the coronary microcirculation in normal and ischemic myocardium. J Cardiovasc Pharmacol 1992; 19(5):755-763. pmid:1381774

27. Wei J, Cheng S, Merz CNB. Coronary microvascular dysfunction causing cardiac ischemia in women. JAMA 2019; 322(23):2334-2335. doi:10.1001/jama.2019.15736

28. Rosen SD, Paulesu E, Wise RJ, Camici PG. Central neural contribution to the perception of chest pain in cardiac syndrome X. Heart 2002; 87(6):513-519. doi:10.1136/heart.87.6.513

29. Knuuti J, Wijns W, Saraste A, et al. 2019 ESC guidelines for the diagnosis and management of chronic coronary syndromes. Eur Heart J 2020; 41(3):407-477. doi:10.1093/eurheartj/ehz425

30. Saraste M, Koskenvuo J, Knuuti J, et al. Coronary flow reserve: measurement with transthoracic Doppler echocardiography is reproducible and comparable with positron emission tomography. Clin Physiol 2001; 21(1):114-122. doi:10.1046/j.1365-2281.2001.00296.x

31. Gagliardi MG, Adorisio R, Crea F, Versacci P, DiDonato R, Sanders SP. Abnormal vasomotor function of the epicardial coronary arteries in children five to eight years after arterial switch operation: an angiographic and intracoronary Doppler flow wire study. J Am Coll Cardiol 2005; 46(8):1565-1572. doi:10.1016/j.jacc.2005.06.065

32. Wei J, Mehta PK, Johnson BD, et al. Safety of coronary reactivity testing in women with no obstructive coronary artery disease: results from the NHLBI-sponsored WISE (Women's Ischemia Syndrome Evaluation) study. JACC Cardiovasc Interv 2012; 5(6):646-653. doi:10.1016/j.jcin.2012.01.023

33. Schindler TH, Schelbert HR, Quercioli A, Dilsizian V. Cardiac PET imaging for the detection and monitoring of coronary artery disease and microvascular health. JACC Cardiovasc Imaging 2010; 3(6):623640. doi:10.1016/j.jcmg.2010.04.007

34. Murthy VL, Naya M, Taqueti VR, et al. Effects of sex on coronary microvascular dysfunction and cardiac outcomes. Circulation 2014 129(24):2518-2527. doi:10.1161/CIRCULATIONAHA.113.008507 


\section{TJOE AND COLLEAGUES}

35. Kodzwa R. ACR manual on contrast media: 2018 updates. Radiol Technol 2019; 91(1):97-100. pmid:31471485

36. Feher A, Sinusas AJ. Quantitative assessment of coronary microvascular function: dynamic single-photon emission computed tomography, positron emission tomography, ultrasound, computed tomography, and magnetic resonance imaging. Circ Cardiovasc Imaging 2017; 10(8):e006427. doi:10.1161/CIRCIMAGING.117.006427

37. Samim A, Nugent L, Mehta PK, Shufelt C, Bairey Merz CN. Treatment of angina and microvascular coronary dysfunction. Curr Treat Options Cardiovasc Med 2010; 12(4):355-364 doi:10.1007/s11936-010-0083-8

38. Pauly DF, Johnson BD, Anderson RD, et al. In women with symptoms of cardiac ischemia, nonobstructive coronary arteries, and microvascular dysfunction, angiotensin-converting enzyme inhibition is associated with improved microvascular function: A double-blind randomized study from the National Heart, Lung and Blood Institute Women's Ischemia Syndrome Evaluation (WISE). Am Heart J 2011; 162(4):678-684. doi:10.1016/j.ahj.2011.07.011

39. Kayikcioglu M, Payzin S, Yavuzgil O, Kultursay H, Can LH, Soydan I. Benefits of statin treatment in cardiac syndrome-X1. Eur Heart J 2003; 24(22):1999-2005. doi:10.1016/s0195-668x(03)00478-0

40. Lee BK, Lim HS, Fearon WF, et al. Invasive evaluation of patients with angina in the absence of obstructive coronary artery disease. Circulation 2015; 131(12):1054-1060. doi:10.1161/CIRCULATIONAHA.114.012636

41. Lerman A, Burnett JC Jr, Higano ST, McKinley LJ, Holmes DR Jr. Long-term L-arginine supplementation improves small-vessel coronary endothelial function in humans. Circulation 1998; 97(21):21232128. doi:10.1161/01.cir.97.21.2123

42. Cannon RO 3rd, Watson RM, Rosing DR, Epstein SE. Efficacy of calcium channel blocker therapy for angina pectoris resulting from small-vessel coronary artery disease and abnormal vasodilator reserve. Am J Cardiol 1985; 56(4):242-246. doi:10.1016/0002-9149(85)90842-2

43. Lanza GA, Maseri A. Coronary artery spasm. Curr Treat Options Cardiovasc Med 2000; 2(1):83-90. doi:10.1007/s11936-000-0031-0

44. Laksanakorn W, Laprattanagul T, Wei J, et al. Cardiac rehabilitation for cardiac syndrome $X$ and microvascular angina: a case report. Int
J Case Rep Images 2015; 6(4):239-244.

45. Bairey Merz CN. Testing for coronary microvascular dysfunction. JAMA 2019; 322(23):2358. Published November 18, 2019. doi:10.1001/jama.2019.16625

46. Sedlak TL, Lee M, Izadnegahdar M, Merz CN, Gao M, Humphries $\mathbf{K H}$. Sex differences in clinical outcomes in patients with stable angina and no obstructive coronary artery disease. Am Heart J 2013; 166(1):38-44. doi:10.1016/j.ahj.2013.03.015

47. Taqueti VR, Solomon SD, Shah AM, et al. Coronary microvascular dysfunction and future risk of heart failure with preserved ejection fraction. Eur Heart J 2018; 39(10):840-849. doi:10.1093/eurheartj/ehx721

48. Mohammed SF, Hussain S, Mirzoyev SA, Edwards WD, Maleszewski JJ, Redfield MM. Coronary microvascular rarefaction and myocardial fibrosis in heart failure with preserved ejection fraction. Circulation 2015; 131(6):550-559. doi:10.1161/CIRCULATIONAHA.114.009625

49. Paulus WJ, Tschöpe C. A novel paradigm for heart failure with preserved ejection fraction: comorbidities drive myocardial dysfunction and remodeling through coronary microvascular endothelial inflammation. J Am Coll Cardiol 2013; 62(4):263-271. doi:10.1016/j.jacc.2013.02.092

50. Owan TE, Hodge DO, Herges RM, Jacobsen SJ, Roger VL, Redfield MM. Trends in prevalence and outcome of heart failure with preserved ejection fraction. N Engl J Med 2006; 355(3):251-259. doi:10.1056/NEJMoa052256

51. Handberg EM, Merz CNB, Cooper-Dehoff RM, et al. Rationale and design of the Women's Ischemia Trial to Reduce Events in Nonobstructive CAD (WARRIOR) trial. Am Heart J 2021; 237:90-103. doi:10.1016/j.ahj.2021.03.011

52. Johnson GL, Henry TD, Povsic TJ, et al. CD34+ cell therapy significantly reduces adverse cardiac events, health care expenditures, and mortality in patients with refractory angina. Stem Cells Transl Med 2020; 9(10):1147-1152. doi:10.1002/sctm.20-0046

Address: Chrisandra Shufelt, MD, MS, Barbra Streisand Women's Heart Center, Cedars-Sinai Smidt Heart Institute, 8631 W. Third Street, Suite 740, Los Angeles, CA, 90048; Chrisandra.Shufelt@cshs.org 